\title{
La importancia del ceremonial en la diplomacia durante el reinado de Felipe $\mathbf{V}$
}
The importance of the ceremonial in diplomacy during the reign of Felipe V

\author{
Julio M Panizo ${ }^{1}$. \\ juliom.panizo@invi.uned.es \\ juliom.panizo@uvic.cat \\ juliom.panizo@upf.edu
}

Recepción: 10/12/17 Revisión: 14/12/17 Aceptación: 14/12/17 Publicación: 18/12/17

\begin{abstract}
Resumen
La relación de las diferentes monarquías con las casas reales de su entorno siempre ha sido un elemento fundamental para el ejercicio de la política exterior, afectando en muchos casos por los vínculos familiares a las relaciones familiares. La presencia de representantes de los demás monarcas en las diferentes cortes desde finales del siglo XV es habitual y el trato que se daba a esos representantes era una forma más de gestionar esa diplomacia de la época.

La disparidad de criterios y costumbres en ese trato con los embajadores hace que en muchas de las cortes se dicten libros de etiquetas en donde se especifica de forma detallada las costumbres a seguir en este trato, así como en la participación de las diferentes ceremonias de la Corte. La llegada de Felipe $V$ al trono, introdujo nuevas costumbres en la monarquía española como fruto de su origen francés y que al igual que afectaron a la actividad de la corte también se visualizaron en esas costumbres con los representantes de otras monarquías.
\end{abstract}

Palabras clave: diplomacia, embajador, ceremonial, Felipe V.

\section{Abstract}

The relationship of the different monarchies with the royal houses of their environment has always been a fundamental element for the exercise of foreign policy, affecting in many cases by family ties to family relationships. The presence of representatives of the other monarchs in the different courts since the end of the 15th century is common and the treatment given to these representatives was one more way of managing that diplomacy of the time.

\footnotetext{
${ }^{1}$ Dr. en derecho y ciencias sociales. Profesor del Máster Universitario en Protocolo de la UNED y del Departamento de comunicación de la UPF, UVIC-UCC
} 
The disparity of criteria and customs in this deal with the ambassador's makes that in many of the courts labels are dictated where the customs to be followed in this deal are specified in detail, as well as in the participation of the different ceremonies of the Cut. The arrival of Felipe $\mathrm{V}$ to the throne, introduced new customs in the Spanish monarchy like fruit of its French origin and that like they affected to the activity of the court also they were visualized in those customs with the representatives of other monarchies.

Keywords: diplomacy, ambassador, ceremony, Felipe V.

\section{Sumario}

1. Introducción.

2. El cambio de dinastía.

3. El ceremonial diplomático.

4. La llegada del embajador.

5. Actos de la vida cotidiana de los embajadores.

6. Conclusiones.

7. Bibliografía

\section{INTRODUCCIÓN.}

En el mundo actual nadie pone en duda el papel de la diplomacia para el buen desarrollo de las relaciones entre Estados y dentro de esta diplomacia existen toda una serie de normas y costumbres que canalizan la forma de gestionar estas relaciones.

En la actualidad el corpus de estas normas está establecido en la Convención de Viena de 1961 sobre las relaciones diplomáticas, suscrito en la actualidad por 191 países, lo que hace que en la actualidad todos los países, aunque no hayan suscrito el mismo regulan sus relaciones diplomáticas en base a los preceptos marcados en este documento(United Nations, 2017).

Pero hace no muchos siglos la forma en la que se desarrollaban las relaciones diplomáticas entre los diferentes reinos, era muy diferente y desarrollaban de forma particular la política exterior del momento, con todas las limitaciones que en ese momento existían, como por ejemplo en el ámbito de las comunicaciones. La ausencia de una normativa de aplicación global hacía que los diferentes reinos establecieran una normativa propia sobre la forma de actuar con los representantes de otros monarcas y que no es ni más ni menos que el reflejo de la forma en que se desarrollaba la diplomacia en ese momento. 
Los libros de etiquetas era la denominación que tenían las recopilaciones de las diferentes regulaciones sobre ceremonial y protocolo que se desarrollaban en torno a la figura del rey y no de los aspectos que aparecen detallados son los que afectan a las diferentes cortesías que se deberían de tener con los ministros o embajadores de otros reyes que forman parte de la estructura de la corte. Estas cortesías no dejan de ser la forma en la que se desarrolla el protocolo y el ceremonial diplomático del momento y que por la evolución histórica con el nacimiento de los Estados modernos y de las comunicaciones y medios de transporte ha desembocado en el protocolo y el ceremonial diplomático actual.

\section{EL CAMBIO DE DINASTÍA.}

La Casa de Habsburgo llegó a España con Carlos de Habsburgo o Carlos I que hereda el reino de Castilla de su madre Juana "la loca" y el reino de Aragón de su abuelo el rey Fernando de Aragón. Pero esta dinastía se extingue con el rey Carlos II conocido como "el hechizado" por las importantes minusvalías que tenía con motivo de la constante consanguineidad de las diferentes generaciones de la familia. Su incapacidad para poder reinar hizo que la gestión del reino estuviese de forma permanente en manos de los cortesanos, especialmente los Grandes de España y gran parte de los nobles. Entre las diferentes incapacidades que tenía estaba la esterilidad lo que le impidió tener hijos y como consecuencia al no haber herederos directos ni indirectos al trono, en el momento de su fallecimiento fue necesario proveer el acceso al trono de un nuevo monarca.

El acceso al trono supuso un fuerte conflicto entre los reinos vecinos y dentro del territorio español para determinar un posible candidato entre las dos dinastías que aspiraban a continuar con la Casa Real de España, la dinastía de los Borbones de origen francés y la dinastía de Austria. Finalmente el nieto del rey Luis XIV de Francia, el candidato de la Casa de Borbón accedió al trono español con el nombre de Felipe V (Garcia Càrcel \& García Cárcel, 2002).

La llegada al trono de Felipe $V$ no se realizó sin conflictos internos en la propia corte, puesto que los nobles que gestionaban de facto la corte durante el reinado de su antecesor, Carlos II, hicieron que en su testamento apareciese de forma expresa que sus nobles deberían de permanecer en los puestos que tenían encomendados hasta ese momento. Situación que en la práctica no se cumplió con el consiguiente malestar. El riesgo de que el nuevo rey fuese controlado por los cortesanos hizo que el embajador de Francia, el duque de Harcourt se encargara de rediseñar la estructura de la nueva Casa del Rey y poder introducir personas de su confianza.

La propia llegada de Felipe $V$ a España ya desarrollada con un premeditado ceremonial, llegando hasta la frontera acompañado de los nobles franceses para ser recibido y acompañado desde la frontera por nobles españoles, en reconocimiento de su status.

Los cambios se iniciaron rápidamente puesto que el rey de Francia no quería que el nuevo rey de España permaneciera recluido en Palacio, como pretendían los nobles españoles para poder controlarlo de la misma forma que habían hecho hasta entonces, sino que se buscaba darle una visibilidad para generar la aceptación por parte de sus súbditos. Es el propio "rey Sol" el que pone como ejemplo de la forma de actuar del nuevo rey que debe de imitar el ejemplo de los reyes de Francia o del mismo Carlos I (Gómez-Centurión Jiménez, 2000). 
El marqués de Villafranca, que ejercía como mayordomo de la Casa del Rey se encargó de generar un informe para la reducción de gastos y de criados entre los oficiales del Palacio.

El ceremonial fue desde el primer momento la clave para generar aprobación por esta nueva dinastía para logara la aceptación del pueblo. Se hicieron ceremonias de presentación pública del nuevo rey en Cataluña y en Nápoles con ceremonias desarrollada según el estilo de la corte francesa. Un ejemplo, que aunque parezca insignificante hoy en día, no lo era en su momento fue el de no querer utilizar cortinas en las ceremonias litúrgicas realizadas en estas presentaciones públicas. Hasta ese momento los monarcas se protegían de la visión de los ciudadanos durante los servicios religiosos con una cortina de la que el "sumiller de cortina" era el máximo responsable.

Pero los cambios fueron más profundas de lo que podría parecer y todos con una fuerte resistencia por el entorno directo del rey. Uno de los que más conflicto generó fue el cambio en las costumbres sobre qué personas tiene acceso al rey dentro del Palacio y que había establecido el rey Carlos de Habsburgo y que generaron gran incomodidad entre los nobles como manifestó el Conde de Fuensalida.

Señor, la Nación Española no admite familiaridades con su Rey, y mucho menos el verse preferidos con desigualdad. Las entradas en el cuarto de V.M. están relajadas con escándalo [...] Esto, Señor, que parece materia gubernativa y que repugna a la libertad común de las gentes, que prescriben los particulares cada uno en su casa que con más razón la debería tener V.M., es materia de gravísimo escrúpulo en el fuero de la conciencia. V.M. tiene jurados a estos Reinos las leyes, fueros, estilos y costumbres, y confirmados todos los honores y dignidades, con que no es de dudar vienen a quedar revocadas las prerrogativas de las dignidades con esta relaxación del Palacio de V.M. (Gómez-Centurión Jiménez, 2000, p. 2122)

Pero hasta en la forma de vestir se van produciendo los cambios introduciéndose poco a poco la moda francesa y desterrando el tradicional traje de golilla y de colores oscuros que se utilizaba en España, no sin fuerte resistencia por gran parte de los miembros de la corte.

Es en el año 1704 cuando se inicia las reformas en profundidad, algunas de las más problemáticas afectaron a los capitanes de la guardia, que desde ese momento sólo reciben órdenes directamente del rey, suponiendo una pérdida de funciones del mayordomo mayor que era el que hasta ese momento transmitía estas órdenes del rey a los capitanes, pero también se les dio un papel importante en los actos públicos puesto que se determinó que se situarían siempre a continuación del monarca sin que hubiera ninguna otra persona entre medio de ambos, ocupando las sillas situadas inmediatamente detrás, lugar en el que se sentaban hasta ese momento los jefe de palacio, salvo que hubiera otra autoridad más importante. Todo esto generó un gran descontento entre los nobles.

Un ejemplo de estos conflictos se desencadenó el día 25 de septiembre de 1705 con motivo de la celebración de un Te Deum con motivo del cumpleaños del rey de Francia. Tradicionalmente los nobles con título de grandeza ocupaban los bancos inmediatos al del 
rey, pero en esta ocasión este banco no se colocó en ese sitio y los asistentes se iban colocando por orden de llegada sin ningún criterio de precedencia que marcase la jerarquía, siendo el capitán de la guarda el único que tenía un puesto reservado detrás del rey. Los nobles se sintieron tan molestos que boicotearon la ceremonia y finalmente tuvo que realizarse en privado y sin público.

Uno de los principales artífices de todos los cambios que se van produciendo, la princesa de los Ursinos, Marie-Anne de La Trémoille. Gozaba de la plena confianza de Luis XIV, por este motivo le encargó acompañar al joven rey Felipe $V$ y se le encargó asumir las funciones de camarera mayor de la reina Maria Luisa Gabriela de Saboya y fue una negociadora fundamental en las segundas nupcias del rey con Isabel de Farnesio.

Entre sus influencias estuvo la de ejercer de facto como maestra de ceremonias de todos los actos que se hacían en la alcoba real y de los criterios necesarios para poder acceder a los aposentos reales. Entre los cambios estuvo la creación de una alcoba compartida por los dos reyes en vez de los dormitorios separados que existían en la Casa de Habsburgo y son sustituidos por hileras de antecámaras previas a los salones reales como era costumbre en la corte francesa. El objetivo fundamental de todos estos cambios en el ceremonial era que el palacio cambiara de concepción y en vez de ser un refugio para el rey fuera un lugar para exponer y dar visibilidad al monarca (Saint_Rene Taillandier, 1926).

Esta exposición del monarca llega hasta el extremo de que los actos más privados del monarca se convierten en actos públicos a los que tienen acceso los cortesanos y los representantes extranjeros como ocurría para el momento en el que se vestía el monarca, acto en el que participaban los mayordomos, los ministros extranjeros y otros personajes de interés en cada momento.

\section{EL CEREMONIAL DIPLOMÁTICO.}

La regulación del ceremonial diplomático en la corte del rey Felipe $\mathrm{V}$ gira en torno a tres líneas fundamentales, la primera de ellas es la constante mezcla existente entre el ceremonial de la antigua práctica que se seguía con la dinastía anterior y las nuevas formas introducidas al estilo de la corte de París. La segunda es la tendencia constante a la apertura de las estancias dentro del Palacio en donde se desarrolla gran parte de la vida diaria del monarca, facilitando así su acceso y convirtiéndose así en un escaparate para el contacto con su entorno. Y por último la figura del conductor de embajadores, que actúa como autentico y constante intermediario entre el monarca y los representantes extranjeros que se encuentran en la corte, de manera que no hay nada que tenga que relación con estos embajadores que no pase por sus manos y no caiga bajo su responsabilidad (Barrios, 1988).

Los embajadores se organizaban en torno a diferentes categorías, en primer lugar el denominado como embajador doméstico que era el embajador del rey de Francia, y que era conocido así por la vinculación familiar que existía entre los dos reyes y que anteriormente, con la dinastía de Habsburgo, ocurría con el representante de la monarquía austríaca, por esta misma razón. Este embajador doméstico tenía una serie de privilegios como el tener mucha más facilidad para acceder a audiencias con el rey de España, siendo recibido el mismo día que llega a la corte si lo solicita. 
Luego están el resto de representantes que se denominaban ministros públicos y que podían tener dos categorías, los ordinarios que compartían de forma habitual la vida de la corte y los extraordinarios que eran enviados para ejercer alguna función especial y su permanencia en la corte era por un tiempo reducido. En el caso de los ministros extraordinarios se les otorgan una serie de privilegios especiales como el poder disfrutar de alguno de los coches de las Caballerías Reales o el ofrecimiento de alojamiento durante un máximo de 9 días por cuenta del Palacio Real.

Se destaca de forma particular en libro de etiquetas a los "Nuncios y embaxadores de Coronas y los de Venecia y Estados Generales" (Felipe V, 1715, p. 6v-7r) a los que se les da un trato preferente en las atenciones lo mismo que ocurre con los denominados como "ministros de capilla", por ser los que pertenecen a monarquías con las que comparte la misma confesión religiosa.

\section{LA LLEGADA DEL EMBAJADOR.}

La recepción pública en la corte de un nuevo embajador es el momento que permite el inicio de sus funciones, pero para ello es necesario que previamente se notifique la fecha de su llegada a la corte indicando día, hora y el recorrido que hará para que el Conductor de Embajadores pueda ir a recibirlo a una aldea cercana. El cargo de Conductor de Embajadores, es el cargo más antiguo de la administración civil española y fue creado por Felipe IV el 1 de abril de 1626 (Luis Ayllón, 2004).

El Conductor ha de solicitar en la Secretaría de Despacho permiso para que la Real Caballería le asigne un coche con caballos para ir a recibir al nuevo embajador, labor que se hacía aproximadamente a unas dos leguas de la corte $(9,6 \mathrm{~km})$ en donde espera a su llegada. En ese coche solamente suben el embajador y el conductor de embajadores que irán seguidos por el resto de la comitiva que acompañe al nuevo representante y se dirigirán todos hacia el lugar de alojamiento previsto. Una vez en sus aposentos un gentilhombre notifica al rey, a la reina y al ministro de estado la llegada.

Una vez en sus aposentos el nuevo embajador entrega al ministro de estado una copia de las cartas credenciales y la explica las funciones que le han sido encargadas durante su estancia en la corte de Madrid para poder notificar el ministro su aceptación y fijar la fecha en la que tendrá lugar la primera audiencia con el rey y en la que le hará entrega de las cartas credenciales.

Por la consideración de ministro doméstico que hemos comentado que tenía el representante del rey de Francia, en su caso la recepción con el rey se hacía el mismo día de su llegada a la corte.

"... se ha de practicar lo mismo que por lo pasado en tiempo del Sor Rey Carlos segundo, y sus Antecessores de la Casa de Austria, se hacía y observaba con el Embajador de Alemania, considerándole como Domestico y así se escusará con el referido Embajador de Francia este cumplimiento, pues cmo de casa podrá el mismo dia que llegáre a la Corte ir en derechura 


\section{a Palacio y tener Audiencia privada de S.M. a diferencia de los otros."(Felipe V, 1715, fol. 4r-5v)}

Una vez que se ha decidido la fecha en la que se hará la recepción por parte del monarca, también conocida como "entrada pública", por ser el acto público oficial de entrada en la corte y que da oficialidad a su actividad como embajador. Es el mayordomo mayor el que notifica al conductor de embajadores el día y la hora en la que tendrá esa entrada y motivo por el cual el conductor de embajadores tendrá que ponerse en contacto con el secretario de despacho del rey para que prepare los caballos necesarios para dicha ceremonia.

El mayordomo mayor de SM el rey, cargo equivalente a lo que hoy sería el jefe de la Casa de SM el rey, junto con el gentilhombre de boca más antiguo, en calidad de decano de la Real Casa y que formaba parte de los cortejos reales en las comidas, en las ceremonias de la capilla y en solemnidades públicas (Real Academia Española (RAE), 2014) se dirigen en coche a la residencia que ocupa el nuevo embajador. Allí el conductor de embajadores instruirá al sobre el desarrollo de la bienvenida al embajador los recibirá en primera grada de la escalera de entrada a la residencia y saluda a todos dándoles la mano.

Se prepara a continuación la comitiva que se dirige al Palacio, en la que los nuevos embajadores no pueden ir acompañados por su familia o compañeros, pero si puede estar formada por otros embajadores y ministros, cardenales, etc. El orden de la comitiva debía de ser:

Coche donde viaja el embajador acompañado por el conductor de embajadores

Coche de SM con la comitiva real

Caballerizo mayor del embajador a caballo retrasado.

La posición del caballerizo mayor del embajador debió de ser causa de conflicto puesto que se menciona expresamente que antes del coche de SM el rey no "se interponga el caballerizo del Ministro, como lo han intentado muchos, aunque no lo ha conseguido ninguno, sino que vaya a un lado, dos o tres passos detrás del caballo de su Amo, como está reglado y se ha observado desde antiguo" (Felipe V, 1715, fol. 10r).

También supuso un conflicto en las entradas públicas de los Nuncios se intentó que los ministros del Tribunal de la Nunciatura se situarán detrás de su coche y por delante del coche de la Casa Real a posición del Nuncio en este tipo de ceremonias puesto que intento en varias ocasiones situarse detrás del coche del embajador, por lo que se determinó que en ningún caso podría situarse nadie entre el coche del embajador y el de los representantes del Palacio Real.

Durante todo el recorrido de la audiencia ha de haber dos filas de alabarderos flanqueando el recorrió hasta la escalera que lleva a la sacristía de la capilla y se mantendrá formada hasta el final de la audiencia.

Al entrar en la estancia el mayordomo mayor y el embajador hacen una reverencia y seguirá acompañado del mayordomo hasta realizar la segunda reverencia, momento en el que el embajador se aproxima al rey para entregar las cartas credenciales tras cubrirse la cabeza por indicación del rey. Al acabar la audiencia ha de retirarse sin dar la espalda al rey y saludando d los nobles que lo acompañan en este acto. 
“... acercandose este a S.M: hasta que media alguna corta distancia, le oye S.M. en pie, y mandandole se cubra, pone en sus R[eale] $]^{5}$ manos la carta original que trae de su Soberano en credencia de su embaxada, y concluida la Audiencia, sale retirándose sinvolver la espalda saludando a los Grandes desde la segunda reverencia" (Felipe V, 1715, fol. 13v-14r).

Una vez terminada la audiencia con el rey se pasa al Cuarto de la Reina en donde tiene audiencia con ella, en la antesala del Cuarto es recibido por el mayordomo semanero y en la puerta de la Cámara por el mayordomo mayor. En esa audiencia tras el besamanos se saluda a las camareras y demás damas de compañía de la reina. A continuación se procede a las audiencias con el resto de miembros de la Familia Real, primero con el heredero en el Cuarto del Príncipe y luego en el Cuarto de los Infantes con el resto de hijos por orden de edad, en todo este proceso siempre acompañado por el mayordomo mayor.

Tras la finalización de las audiencias el embajador se dirige a su residencia siendo acompañado por las mismas personas que lo acompañaron en su camino hacia el Palacio Real, el mayordomo mayor, el gentilhombre de boca y el conductor de embajadores, en donde es despedido en la misma puerta, al igual que se hizo al recibirlos.

La tarde del mismo día en el que tiene lugar la entrada pública o audiencia en el Palacio Real el embajador ha de visitar al ministro de Estado que le devolverá la visita posteriormente siendo el conductor de embajadores el que se encargará de dar las instrucciones para el correcto desarrollo de la visita.

\section{ACTOS DE LA VIDA COTIDIANA DE LOS EMBAJADORES.}

Siempre que un ministro extranjero quiera solicitar audiencia con el rey ha de tramitar esa petición siempre a través del conductor de embajadores. Cuando estas audiencias son para las entradas públicas o lo que hoy llamamos presentación de cartas credenciales la petición de audiencia se hace a través del mayordomo mayor, en el resto de los casos, las llamadas "audiencias de cumplimiento" la petición se tramita a través del secretario de la Cámara. Si la audiencia se pide con la reina la petición de la audiencia ha de tramitarla el conductor de embajadores a través de la camarera mayor y siempre que previamente haya tenido audiencia con el rey.

Solo cuando haya un día festivo, denominados como "días de joya", es posible tener una audiencia sin la formalidad de una petición previa, en esas ocasiones se convoca a los representantes extranjeros en la antecámara de embajadores, aunque solamente podrán participar los que hayan hecho la entrada pública.

Con motivo de celebraciones particulares como cumpleaños, celebración de festividades religiosas como la Navidad, Reyes o Resurrección se estableció una nueva forma de actuar en la que se invita por escrito a todos los denominados embajadores de capilla a participar en la ceremonia religiosa concediéndosele después audiencia pública. Anteriormente sólo se invitaba al Nuncio o al embajador de la Orden de Malta. Entre estas posibles celebraciones se introduce una nueva modifación y es el no conceder audiencias en los días de las 
onomásticas de los miembros de la Familia Real, por lo que se pide que se notifique al conductor de embajadores y a todos los responsables de las casa reales extranjeras.

En el caso de que se concedan audiencia a representantes de monarcas no católicos estas se harán por la tarde permitiendo que los miembros de la Familia Real puedan participar de la ceremonia religiosa por la mañana.

"Que a los Embaxadores y Ministros que no fueren de Principes Catholicos se les señalara por la tarde, en observancia de la antigua práctica o estilo, las Audiencias que pidieren para la ceremonia ade los días festivos y cumplimiento de años y Pasquas, y no para negocio de su Ministerio" (Felipe V, 1715, fol. 19v-20r)

Pero la presencia en la Corte de los ministros extranjeros es muy habitual en otros momentos fuera de las tradicionales audiencias y de los momentos de celebración y en algunos casos con importantes cambios en el estilo. El acceso al Cuarto del rey se permite a partir de ahora a los ministros extranjeros en donde se podía presenciar el momento del vestido y desvestido del rey, al igual que hacían los criados del Palacio, pero siempre con la condición de que en esos momento no se podría hablar de negocios, puesto que para eso sería imprescindible la solicitud de audiencia. También se recupera la antigua costumbre de que el rey invite a los embajadores de capilla a las comedias del Buen Retiro y los demás festejos del Palacio. En todos estos casos los embajadores serán recibidos por el conductor de embajadores en el primer tramo de la escalera que los acompañará hasta donde han de tomar las carrozas que los trasladarán hasta el lugar del festejo.

En las celebraciones en el Buen Retiro se prepara un sito para todo los embajadores y otro especial para el Nuncio en el caso de que fuera cardenal indicándoles por medio del conductor de embajadores que han de recoger "las boletas" avisándoles que han de lleva bancos o taburetes.

Cuando las celebraciones son máscaras u otros festejos que pasen por la plaza del Palacio se les reservará a los embajadores "el balconcillo baxo contiguo a la Puerta principal entrando por ella a mano izquierda"

De forma similar se gestiona la invitación de los embajadores a las fiestas de los toros en los que el conductor ha de avisar al mayordomo mayor de los embajadores que acudirán para asignarles los balcones desde lo que podrán seguir el festejo con el único requisito de que no podrán poner en los balcones ningún tipo de adorno que pueda parezcan un dosel, puesto que sólo el balcón donde se sitúa el rey puede tenerlo.

El 14 de abril de 1701 fecha en la que el rey Felipe $V$ hizo la entrada pública en la corte, los embajadores excusaron su asistencia alegando como justificación que no se les permitió la posibilidad de ir inmediatamente detrás del rey en la comitiva a caballo, puesto que se decidió que detrás debía de ir siempre el caballerizo mayor. Este cargo era uno de los cargos más importantes de la estructura del Palacio y que además de la gestión de la caballeriza acompañaba al rey en todas las salidas fuera del Palacio. Esta situación hizo que en la regulación de las diferentes ceremonias públicas en las que la comitiva se desplaza a caballo 
y en las que acudan los embajadores se determinó cual es el lugar que les corresponde a estos quedando determinada la precedencia de la forma siguiente:
Rey.
Caballerizo mayor.
Mayordomo mayor.
Sumiller de corps.
Capitán de la guardia.
Embajadores

Existe regulada una ceremonia muy especial destinada en exclusiva a los embajadores de Venecia, según la cual cuando estos terminan sus funciones en la corte piden al rey que los arme caballeros en el caso de que no hayan sido armados por parte de otro monarca. Para ello se sigue de forma estricta la ceremonia de la Caballería Mayor en la que se detallan los diferentes pasos del ceremonial al a seguir en el que la espada, como es tradicional, juega un papel muy importante como símbolo.

\begin{abstract}
"...se reducen a recibirlos S.M. sentado. Que el embaxador hechas las reverencias, se pone de rodillas sobre una almohada, sin cubrirse antes ni después; y el Caballerizo Mayor toma el estoque (que está prevenido) con la mano izquierda por el puño y con la derecha por la cuchilla. Que S. M. le recibe, y tocando con él al embaxador en los dos hombros, le dice: Dios os haga buen Cavallero, y se le dá después á besar." (Felipe V, 1715, fol. 25r)
\end{abstract}

El final del reinado.

Al final del reinado y apenas dos años antes de decidir abdicar se producen importante cambios en el ceremonial tal y como lo hemos expuesto. Los frecuentes casos de crisis de ansiedad y de pánico sufridos por el monarca en diferentes actos públicos fueron provocando que el rey poco a poco fuera aislándose más de la presencia pública y permaneciendo cada vez, mas tiempo en el palacio y aislado de la corte. Este aislamiento llego a un punto tal en el que permanencia prácticamente recluido en sus aposentos sin tener apenas contacto ningún con el exterior ni con los miembros de la corte.

Esta situación del rey fue haciendo que las ceremonias iban poco a poco perdiendo importancia llegando a una situación de casi ausencia total de ceremonias en la corte, o las pocas que había perdían todo tipo de solemnidad, como ocurrió con las entradas públicas de los representantes diplomáticos que entregaban sus cartas credenciales de forma totalmente informal y sin prácticamente ningún resto de la formalidad que se había establecido inicialmente en el libro de etiquetas de la corte.

\title{
6. CONCLUSIONES.
}

A la vista de la información presentada podemos concluir que el ceremonial ha sido siempre una herramienta de comunicación al servicio del poder, como lo muestra el hecho de que la llegada de Felipe $V$ a España suponía un intento de cambiar las costumbres de la Casa de Habsburgo para introducir las costumbres francesas de la Casa de Borbón. El ceremonial 
establecido fue uno de los principales elementos comunicativos para la introducción de ese estilo más abierto y que diera más visibilidad al rey frente a la corte y el resto de súbditos.

Los diferentes cambios en las dinastías y a su forma de entender la manera en la que ejercerán su cargo también generan modificaciones en el ceremonial del momento para poder transmitir de forma más efectiva ese carácter que cada uno de los monarcas quería imprimir a su reinado.

El ceremonial se convierte también en una forma clara de visualizar la forma en la que se ostenta el poder por parte de los monarcas, como hemos visto que de los años iniciales del reinado de Felipe $\mathrm{V}$ a los últimos años se pasó de un ceremonial muy marcado y con un objetivo de visualizar al monarca muy claro a un ceremonial casi inexistente $y$ completamente informal ante el asilamiento del rey.

El ceremonial se muestra como una herramienta fundamental para el ejercicio de las relaciones diplomáticas de la época y que se desarrollaban fundamentalmente con los representantes de otros monarcas que estaban en la corte y de los que dependían los acuerdos y alianzas futuros y que en la corte tenia el carácter de representante del propio monarca al ser la persona que ha designado para que ejerza esa función

\section{BIBLIOGRAFÍA}

Barrios, F. (1988). Práctica diplomática de la corte de España a principios del siglo XVIII: notas de un reglamento de ceremonial de 1717. Revista de Estudios Políticos, 62, 163183.

Felipe V. (1715). Reglamento ceremonial que desde ahora en adelante han mandado SM se ovserve con todos los Ministros Públicos de Principes Extranjeros que vinieron a esta Corte de cualquier grado y Calidad que sean. (Biblioteca Naciona de España, Ed.) (Mss 10411). Madrid.

Garcia Càrcel, R., \& García Cárcel, R. (2002). Felipe V y los españoles : una visión periférica del problema de España. Barcelona: Plaza \& Janés.

Gómez-Centurión Jiménez, C. M. (2000). La corte de Felipe V: el ceremonial y las casa reales durante el reinado del primer Borbón. XIV Coloquio de Historia Canario-Americana, 14(14), 2113-2141.

Luis Ayllón. (2004). El Gobierno pone fin a 376 años de la figura del Introductor de Embajadores | Nacional | Política - Abc.es. Retrieved December 4, 2017, from http://www.abc.es/hemeroteca/historico-21-04-2004/abc/Nacional/el-gobierno-ponefin-a-376-años-de-la-figura-del-introductor-de-embajadores_9621077224976.html 
Real Academia Española (RAE). (2014). Diccionario de la lengua española.

Saint_Rene Taillandier, M. (1926). La princesse des ursins. (Hachette, Ed.).

United Nations. (2017). Vienna Convention on Diplomatics Relations. Retrieved October 31, 2017, from http://legal.un.org/avl/ha/vcdr/vcdr.html 